\title{
ZOOPLANKTON COMMUNITY OF THE EGYPTIAN MEDITERRANEAN COAST
}

\author{
Nagwa E.M. Abdel-Aziz and Sawsan M. Aboul-Ezz \\ National Institute of Oceanography and Fisheries, Alexandria, Egypt.
}

Key words: zooplankton- Egyptian Mediterranean Coast - vertical distribution

\begin{abstract}
The zooplankton community during the present study ( autumn 2000 - winter 2001 ) appeared to has low diversity as compared to that found in the whole Egyptian Mediterranean waters. The western side was characterized by higher diversity than the eastern side, which is affected by land based effluents entering the sea from the coastal lakes and the River Nile. The standing crop values indicated poor zooplankton population in the whole study area. The dominance of species showed drastic changes as compared to the previous studies, whereas the previously dominant species are ranked recently as rare species. The identified species exhibited different seasonal patterns of vertical distribution but the majority of them were recorded in the subsurface layer down to $200 \mathrm{~m}$ depth.
\end{abstract}

\section{INTRODUCTION}

Since the construction of the Aswan High Dam in 1965 and the drop of the amount of the discharged fresh water due to cessation of the Nile flood, the biological and ecological characteristics of the southeastern Mediterranean waters experienced drastic changes. However, the increase in volume of waste waters reaching the neritic area from the coastal lagoons induced more changes in the water quality and biotic components of the ecosystem in this area. Zooplankton represents one of these components which experienced long term variations in its quantitative and qualitative structure. This community in the offshore Egyptian Mediterranean waters has received but little attention (Dowidar, 1974 \& 1988; Hussein, 1977; Anonymous, 1979).

The present paper aims to follow up changes in the dynamics of zooplankton community along the southeastern Mediterranean, 
including standing crop, species composition, dominance of species and their vertical distribution, particularly in offshore water.

\section{MATERIAL AND METHODS}

Zooplankton samples were collected during autumn 2000 and winter 2001 at four sectors, extending offshore perpendicularly to the coast line in front of Sidi Barrani, Matrouh, Alamain and Sahl El-Tina (Fig. 1). From each section, samples were collected through vertical hauls at three to four stations within depth ranges of $25-0 \mathrm{~m}, 50-25 \mathrm{~m}$, $100-50 \mathrm{~m}$ and $200-100 \mathrm{~m}$, using plankton net with diameter of $70 \mathrm{~cm}$ and mesh size of $55 \mu \mathrm{m}$. The samples were concentrated to $100 \mathrm{ml}$ and preserved in $4 \%$ neutralized formalin. The obtained species were identified according to Rose (1933); Tregouboff and Rose (1957); Edmondson (1959); Marshall (1969); Bradford (1972) and Malt (1983). Three aliquots of $5 \mathrm{ml}$ were counted from each concentrated sample and their average was used in calculating the standing stock of zooplankton. The collection of zooplankton samples was carried out throughout the research plan of the National Institute of Oceanography and Fisheries for oceanographic survey of the Egyptian Mediterranean coast.

\section{RESULTS AND DISCUSSION}

The present study recorded relatively low diversity of zooplankton (214 species) in coastal area as compared to the total diversity ( 498 species ) known in the whole Egyptian Mediterranean (Abdel-Aziz, 2002). The spatial distribution of species indicates that the western side of the Egyptian Mediterranean coast harboured more diversified community ( $101-142$ species) than the eastern part (63 species). The low number of species in the eastern part may be attributed to the effect of the land-based effluents discharged to the sea off the Nile Delta Region and also to the low number of samples collected from the eastern part. On the other hand, the autumn community at the different sampling sections was represented by markedly higher number of species (47-129 species) than the late winter (26-82 species). From Table 1, it is observed that 33 species are known to the whole area; 16 were restricted to the western part, while 10 species were found only in the eastern part. In the meantime, 
the rest of species were found sporadically in one or two sampling sections. The species composition demonstrated copepods as the major diversified group in the whole area, represented by 71 species. followed by tintinnids ( 37 species) and radiolaria (18 species). Both copepods and tintinnids included species, which were found in both the near shore and offshore stations, while all radiolarian species were restricted to the offshore stations. The remaining identified species (88 species) belong to many zooplankton groups (Table 1). The relative abundance of zooplankton groups showed different patterns in various sectors (Fig. 2), where the total copepods formed $63 \%$ $92.1 \%$ of total zooplankton in the four sectors, while their contribution decreased to $56.3 \%-87.2 \%$ during late winter. Tintinnids constituted $2-3.3 \%$ during autumn, and increased to $1.3-11 \%$ during late winter. In the meantime, meroplanktonic forms were represented by high percentages (2-16.3\%) during autumn, and (5.1-15.5\%) in late winter. Among copepods, Acartia and Corycaeous attained the highest number of species ( 5 and 7 respectively), while several other genera were represented by 3 species. Meanwhile, the most diversified tintinnid genus was Tintinnopsis ( 7 species) and that of larvaceans was Oikopleura ( 7 species).

Since copepods were the major zooplankton component, their vertical distribution was considered in order to map depth of occurrence for each species (Fig 3). During autumn, 13 species were restricted to the uppermost $50 \mathrm{~m}, 30$ species in the depth range 25 $100 \mathrm{~m}$ and 15 species extended their occurrence to deeper than $100 \mathrm{~m}$. During late winter, 14 species were recorded in the uppermost $50 \mathrm{~m}, 9$ species down to $100 \mathrm{~m}$ and 6 species from $100-150 \mathrm{~m}$ while 12 species appeared in different layers of the water column. From the vertical distribution of copepod species, it could be assumed that several species are considered as shade species, since they are mostly found at depths greater than $100 \mathrm{~m}$, while the depth of euphotic zone in the southeastern Mediterranean was reported to have a maximum of $70 \mathrm{~m}$ in summer (Dowidar, 1988). These species are Candacia bispinosa, Clytemnestra scutellata, Copilia quadrata, Corycaeus limbatus, Eutideus giesbrechti, Corycaeus typicus, Corycella carinata, Corycella rostrata, Eucalanus crassus, Euchaeta spinosa, Lucicutia clavicornis, Macrosetella gracilis, Mecynocera clausi, Paracalanus Pygmaeus, Spinocalanus abyssalis. This is in agreement with Halim (1969) and Weikart (1980 and 1987) who suggested that copepods 
escape to the deeper layers avoiding high temperature at the surface in summer.

The autumn population was dominated by few numbers of species along the whole Egyptian coast, namely, Euterpina acutifrons, Oithona nana, Oithona plumifera Paracalanus parvus and Eucalanus attenuatus. The late winter population in the sector of Sidi Barrani was dominated by the tintinnid Epiplocylis blanda, the copepods Calocalanus pavo, Eucalanus attenuatus, Microsetella rosea, Oithona nana, Oithona plumifera and Paracalanus parvus, the cladocerans Evadne spinifera and the pteropod Limacina inflata. In Alamain sector, the dominant winter forms were the copepod species Eucalanus attenuatus, Oithona nana, Oithona plumifera and Paracalanus parvus and cladoceran Evadne spinifera. The Matrouh sector was dominated by the copepods Paracalanus parvus, Oithona nana, Oithona plumifera, Eucalanus attenuatus, Calanus brevicornis and the protozoans Globigerina ides and Favella ehrenbergii. The winter community in the eastern sector (Sahl El-Tina) showed the following dominant species, Oithona nana, Oithona plumifera Paracalanus parvus, Calocalanus pavo and Clausocalanus arcuicornis. But it is to be noted that the abundance of all the above mentioned species was mostly low relative to the population density of the total zooplankton in the whole area. Remarkably low abundance of zooplankton was found during the present study, whereas the autumn population density varied along the whole coast between 644 indiv. $/ \mathrm{m}^{3}$ at Alamain and 1104 indiv. $/ \mathrm{m}^{3}$ at Sahl El-Tina and the late winter population between 156 and 1316 indiv. $/ \mathrm{m}^{3}$ in the same sectors respectively. Matrouh and Sidi Barrani sectors were inhabited by 647 and 938 indiv. $/ \mathrm{m}^{3}$ during autumn and 252 and 382 indiv. $/ \mathrm{m}^{3}$ during late winter respectively. On the spatial scale, the sector of Sahl- El-Tinah was inhabited by relatively high zooplankton population in autumn as compared to other sectors. This means that the fresh water discharge from Lake Manzalah to the Mediterranean coast may affect the plankton productivity in Sahl EL-Tinah. Dowidar (1988) attributed the reduction of zooplankton stock off the Nile Delta in the period from 1970 to 1985 to the heavy grazing by various planktivores. This explanation contradicts with the pattern of the variations of both sardine catch and zooplankton stock during that period, where the catch of sardine showed a gradual decrease during the period from 1979 to 1983 parallel to the similar decrease in zooplankton abundance during the same period. This means that the 
decrease of zooplankton production may be attributed to ecological factors rather than the effect of intensive grazing by sardine.

It is to be noted that scarce information is so far available about the abundance and structure of zooplankton in the offshore waters of the Egyptian Mediterranean coastal waters. The only known work is that of Anonymous (1979) regarding the zooplankton along the Egyptian Coast from Rosetta to Salloum, but the sampling sites were different from those in the present study which render the comparison between the two studies difficult. However, two sections are yet common among the two studies, upon which comparison could be made. It accordingly seems that the zooplankton standing crop has dropped markedly in the present study as compared to Anonymous (1979). This may be related to the cessation of the Nile flood to the Egyptian Mediterranean coast, which previously promoted high phytoplankton production and consequently high zooplankton count. However, the relative abundance of the existing groups showed no significant difference between the two records as copepods are still the most dominant group (More than $80 \%$ of the total count).

The long-term comparison of zooplankton composition indicates that Paracalanus and Oithona appeared to be the dominant genera during the past three decades in the Egyptian Mediterranean waters, despite the drop of their count recently. In addition, the dominance of species experienced clear variation during the past two decades. According to Dowidar (1988), the following species were dominant in the community of (1984), Acartia clausi, Acarlia latisetosa, Euterpina acutifrons, Centropages violaceous, Isias clavipes, Temora stylifera, Paracalanus aculeatus, Mecynocera clausi, Pleuromamma spp, while all of these species were either rare or completely missing from records of the present study.

\section{REFERENCES}

Abdel-Aziz, N. E. M. (2002). Zooplankton biodiversity in the Egyptian waters of the Mediterranean Sea. Review, Nat.Inst. Oceanogr. \& Fisher.,Egypt. 
Anonymous, (1979). Fisheries investigations of the sardines and other pelagic fish along the Egyptian Mediterranean coast from Rashid to Salloum. II-Biological investigations, Tech. Rep. No $2 / 2$.

Bradford, J. M. (1972). Systematic and ecology of New Zealand central east coast plankton sampled at Kaikoura- New Zeland Oceanographic Institute, No. 54.

Dowidar, N. M. (1974). The phytoplankton of the Mediterranean waters of Egypt. Bull. Nat. Inst. Oceanogr. \& Fisher.,Egypt, $4: 321-342$.

Dowidar, N. M. (1988). Productivity of the Southeastern Mediterranean. M. I. EL-Sabh \& T. S. Murty (eds.), Natural and Man-Made Hazards: 477-498.

Edmondson, W. T. (1959). Freshwater biology, 2nd edition, John Wiley \& Sons Inc. NewYork.

Halim, Y. (1969). Plankton of the Red Sea. Oceanogr. \& Mar. Biol., $7: 231-275$.

Hussein, M. M. (1977). A study of zooplankton in the Mediterranean waters off the Egyptian coast during 1970-1971, with special reference to copepods. M.Sc., Fac. Sci., Alex. Univ., 269 pp.

Malt, S. J. (1983). Fishes distribution du zooplancton. Fixhe No. 169/170/171, Crustacea,Copepoda (Cyclopoida).

Marshall, S. M. (1969). Protozoa. In: Fiches d'identification du zooplancton (Eds. J.H. Fraser de la Mer, Charlottenlund Slot-Danemark.

Rose, M. (1933). Copepods pelagiques, Faune de France, 26: 1-374, la chevalier, Paris. 
Tregouboff, G. and Rose, M. (1957). Manual de Planctologie Mediterranee. C.N.R.S., Paris.

Weikert, H. (1980). On the plankton of the central Red Sea. A first synopsis of results obtained from the cruises MESEDA I \& MESEDA II. Proceedings of Symposium on coastal and Marine Environment of the Red Sea, Gulf of Aden and Tropical Western Indian Ocean. Khartoum, 9-15 January, 1980. The Red Sea and Gulf of Aden Environmental Program, Jeddah: 135-167.

Weikert, H. (1987). Plankton and Pelagic environment in Edwards, A. J., Head, S.M. (eds.) Red Sea. Key Environments. Pergamon Press, Oxford: $90-111$. 
98 Nagwa, E.M. Abdel-Aziz and Sawsan M. Aboul-Ezz

Table 1- Distribution of zooplankton species along the coastal area of the Egyptian Mediterranean waters.

Sidi Barrani Matrouh Alamain Sahl Al-Tina A 00 W01 A 00 W01 A00 W01 A00 A00

\section{Ciliata}

Difflugia lebes

Paramaecium sp.

$\begin{array}{llllllll}0 & 0 & 0 & 0 & 0 & 0 & 1 & 0 \\ 0 & 0 & 0 & 0 & 0 & 0 & 0 & 1 \\ & & & & & & & \\ 0 & 0 & 1 & 0 & 0 & 0 & 0 & 0 \\ 0 & 1 & 1 & 0 & 1 & 0 & 0 & 0 \\ 0 & 0 & 0 & 0 & 0 & 1 & 0 & 0 \\ 0 & 0 & 0 & 0 & 1 & 1 & 0 & 0 \\ 1 & 0 & 0 & 0 & 0 & 0 & 1 & 0 \\ 0 & 0 & 0 & 0 & 1 & 0 & 0 & 0 \\ 0 & 0 & 1 & 0 & 0 & 0 & 0 & 0 \\ 0 & 0 & 1 & 0 & 1 & 0 & 0 & 0 \\ 1 & 0 & 1 & 0 & 1 & 1 & 1 & 0 \\ 0 & 0 & 1 & 0 & 0 & 1 & 0 & 1 \\ 0 & 1 & 1 & 1 & 1 & 1 & 0 & 1 \\ 0 & 0 & 1 & 0 & 1 & 0 & 1 & 0 \\ 0 & 0 & 1 & 0 & 1 & 1 & 0 & 0 \\ 0 & 1 & 0 & 0 & 0 & 0 & 0 & 0 \\ 0 & 0 & 0 & 0 & 1 & 0 & 0 & 0 \\ 0 & 1 & 1 & 1 & 0 & 0 & 0 & 1 \\ 0 & 1 & 0 & 0 & 0 & 0 & 0 & 0 \\ 0 & 0 & 1 & 0 & 1 & 0 & 0 & 0 \\ 1 & 0 & 0 & 0 & 0 & 0 & 0 & 0 \\ 0 & 1 & 1 & 0 & 0 & 0 & 0 & 0 \\ 0 & 0 & 1 & 0 & 0 & 0 & 0 & 0 \\ 0 & 0 & 1 & 0 & 0 & 0 & 0 & 0 \\ 0 & 0 & 1 & 1 & 1 & 0 & 0 & 0 \\ 0 & 0 & 1 & 0 & 0 & 0 & 0 & 0 \\ 0 & 0 & 1 & 0 & 1 & 0 & 1 & 0 \\ 0 & 1 & 0 & 0 & 1 & 1 & 0 & 0 \\ 0 & 0 & 1 & 0 & 1 & 0 & 0 & 0 \\ 0 & 0 & 0 & 0 & 1 & 0 & 0 & 0 \\ 1 & 0 & 0 & 0 & 0 & 0 & 0 & 0 \\ 1 & 0 & 0 & 0 & 0 & 0 & 0 & 0\end{array}$

\section{Tintinnidae}

Amplectella collaria

Codonella aspera

Codonella galea

Codonellopsis longa

Codonellopsis morchella

Codonellopsis onthoceras

Coxliella ampla

Coxliella luciniosa

Epiplocylis blanda

Epiplocylis undella

Eutintinnus fracknoi

Eutintinnus /usus-undae

Eutintinnus macilentus

Favella adriatica

Favella composita

Favella ehrenbergii

Melacylis mediterranean

Metacylis mereschkowskii

Parundella difficilis

Parundella lachmanni

Proplectella angusta

Proplectella pentagona

$\begin{array}{llllllll}0 & 0 & 0 & 0 & 0 & 0 & 1 & 0 \\ 0 & 0 & 0 & 0 & 0 & 0 & 0 & 1 \\ & & & & & & & \\ 0 & 0 & 1 & 0 & 0 & 0 & 0 & 0 \\ 0 & 1 & 1 & 0 & 1 & 0 & 0 & 0 \\ 0 & 0 & 0 & 0 & 0 & 1 & 0 & 0 \\ 0 & 0 & 0 & 0 & 1 & 1 & 0 & 0 \\ 1 & 0 & 0 & 0 & 0 & 0 & 1 & 0 \\ 0 & 0 & 0 & 0 & 1 & 0 & 0 & 0 \\ 0 & 0 & 1 & 0 & 0 & 0 & 0 & 0 \\ 0 & 0 & 1 & 0 & 1 & 0 & 0 & 0 \\ 1 & 0 & 1 & 0 & 1 & 1 & 1 & 0 \\ 0 & 0 & 1 & 0 & 0 & 1 & 0 & 1 \\ 0 & 1 & 1 & 1 & 1 & 1 & 0 & 1 \\ 0 & 0 & 1 & 0 & 1 & 0 & 1 & 0 \\ 0 & 0 & 1 & 0 & 1 & 1 & 0 & 0 \\ 0 & 1 & 0 & 0 & 0 & 0 & 0 & 0 \\ 0 & 0 & 0 & 0 & 1 & 0 & 0 & 0 \\ 0 & 1 & 1 & 1 & 0 & 0 & 0 & 1 \\ 0 & 1 & 0 & 0 & 0 & 0 & 0 & 0 \\ 0 & 0 & 1 & 0 & 1 & 0 & 0 & 0 \\ 1 & 0 & 0 & 0 & 0 & 0 & 0 & 0 \\ 0 & 1 & 1 & 0 & 0 & 0 & 0 & 0 \\ 0 & 0 & 1 & 0 & 0 & 0 & 0 & 0 \\ 0 & 0 & 1 & 0 & 0 & 0 & 0 & 0 \\ 0 & 0 & 1 & 1 & 1 & 0 & 0 & 0 \\ 0 & 0 & 1 & 0 & 0 & 0 & 0 & 0 \\ 0 & 0 & 1 & 0 & 1 & 0 & 1 & 0 \\ 0 & 1 & 0 & 0 & 1 & 1 & 0 & 0 \\ 0 & 0 & 1 & 0 & 1 & 0 & 0 & 0 \\ 0 & 0 & 0 & 0 & 1 & 0 & 0 & 0 \\ 1 & 0 & 0 & 0 & 0 & 0 & 0 & 0 \\ 1 & 0 & 0 & 0 & 0 & 0 & 0 & 0\end{array}$

Rhabdonella amor

Rhabdonella conica

Rhabdonella elegans

Rhabdonella spiralis

Salpingella attenuata

Tintinnopsis brandti

Tintinnopsis compressa

Tintinnopsis cyathus 
Table 1-Continued

Sidi Barrani Matrouh Alamain Sahl Al-Tina

Tintinnopsis cylindrica

Tintinnopsis lindeni

Tintinnopsis lobiancoi

Tintinnopsis radix

Undella hyalina

Undellopsis attenuata

Undellopsis subangulata

\section{Radiolaria}

Acanthosphaera sp

Aulacantha scolymantha

Cladococcus cervicornis

Corocalyptera sp.

Cyphonium ceralospyris

Dictyocephalus mediterranean

Heliosphaera echinoids

Lithostrobus conulus

Pteroscenium pennatum

Sethocapsa pyriformis

Sphaeronectes irregularis

Sphaerozoum punctatum

Spongodictyon trigonizon

Spongotrochus brevispinus

Staurosphaera jacobi

Stylochlamydium asteriscus

Stylocheiron abbreviatum

Theoconus zancleus

\section{Acantharia}

Acanthothiaema robescens

Sphaerocapsa of Gigartacon fragilis

A 00 W of A 00 W ol A 00 W 01 A 00 A 00

\section{Foraminifera}

Adelosina elegans

Ammonia beccari

Globigerina bulloids

Globigerina ides

Globigerina inflala

Globigerinojdes conglobata

Globorotalia truncatuloides

$\begin{array}{llllllll}0 & 1 & 0 & 0 & 0 & 0 & 0 & 0 \\ 0 & 0 & 1 & 0 & 0 & 0 & 0 & 0 \\ 0 & 1 & 0 & 0 & 0 & 0 & 1 & 1 \\ 0 & 1 & 0 & 0 & 0 & 0 & 0 & 0 \\ 1 & 0 & 0 & 0 & 0 & 0 & 1 & 0 \\ 0 & 0 & 1 & 0 & 1 & 1 & 0 & 0 \\ 0 & 1 & 0 & 0 & 0 & 0 & 0 & 0\end{array}$

$\begin{array}{llllllll}0 & 0 & 1 & 0 & 1 & 1 & 0 & 0 \\ 0 & 0 & 1 & 0 & 0 & 0 & 0 & 0 \\ 0 & 0 & 0 & 0 & 1 & 0 & 0 & 0 \\ 0 & 0 & 0 & 0 & 1 & 0 & 0 & 0 \\ 0 & 0 & 1 & 0 & 1 & 0 & 0 & 0 \\ 0 & 1 & 0 & 0 & 1 & 0 & 0 & 0 \\ 0 & 0 & 1 & 0 & 1 & 0 & 1 & 0 \\ 0 & 0 & 0 & 0 & 1 & 0 & 0 & 0 \\ 0 & 0 & 0 & 0 & 1 & 0 & 0 & 0 \\ 0 & 0 & 0 & 0 & 1 & 0 & 0 & 0 \\ 0 & 0 & 0 & 0 & 0 & 0 & 1 & 0 \\ 0 & 0 & 0 & 0 & 1 & 0 & 0 & 0 \\ 0 & 0 & 1 & 0 & 0 & 0 & 0 & 0 \\ 1 & 1 & 1 & 0 & 1 & 1 & 1 & 0 \\ 1 & 0 & 1 & 0 & 0 & 0 & 0 & 0 \\ 0 & 0 & 1 & 0 & 1 & 0 & 0 & 0 \\ 0 & 0 & 0 & 0 & 1 & 0 & 0 & 0 \\ 0 & 1 & 0 & 0 & 1 & 1 & 0 & 0\end{array}$

$\begin{array}{llllllll}0 & 0 & 1 & 0 & 0 & 0 & 0 & 0\end{array}$

$\begin{array}{llllllll}0 & 0 & 1 & 0 & 0 & 0 & 0 & 0\end{array}$

$\begin{array}{llllllll}0 & 0 & 0 & 0 & 0 & 0 & 0 & 1\end{array}$

$\begin{array}{llllllll}0 & 0 & 0 & 0 & 0 & 0 & 0 & 1\end{array}$

$\begin{array}{llllllll}0 & 0 & 0 & 0 & 0 & 0 & 1 & 0\end{array}$

$\begin{array}{llllllll}0 & 1 & 1 & 1 & 1 & 1 & 1 & 0\end{array}$

$\begin{array}{llllllll}1 & 1 & 1 & 0 & 1 & 1 & 0 & 1\end{array}$

$\begin{array}{llllllll}0 & 0 & 1 & 0 & 0 & 0 & 0 & 0\end{array}$

$\begin{array}{llllllll}0 & 1 & 0 & 0 & 1 & 0 & 0 & 1\end{array}$ 
Table 1-Continued

Sidi Barrani Matrouh Alamain Sahl Al-Tina

Orbulina universa

Quiqueloculina striata

Spirilina vivipara

A 00 W 01 A 00 W 01 A 00 W 01 A 00 A 00

Rotifera

Colourella adriatica

Lecane luna

Polyarthra vulgaris

Coelenterata

Abylopsis tetragona

Aglaura haemistoma

Cheloptyes appendicularia

$\begin{array}{llllllll}0 & 0 & 0 & 0 & 0 & 1 & 0 & 0\end{array}$

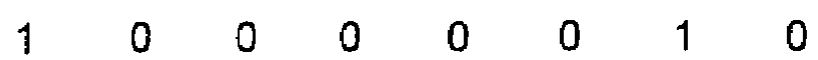

$\begin{array}{llllllll}0 & 0 & 0 & 0 & 0 & 0 & 0 & 1\end{array}$

Chelophyes contorta

Cucullus campanula

Ectopleura dumortieri

Ersaea lessoni

Eudoxoides spiralis

Geryonia proloecidelis

Hippopodius hippopus

Lensia campanella

Lensia conoidea

Lensia multicristata

Lensia subtilis

Liriope tetraphylla

Muggiaea kochia

Obelia spp.

Solmundella bitntaculata

Sphaeronectus irrigularis

Sulculeolaria biloba

$\begin{array}{llllllll}0 & 0 & 0 & 0 & 0 & 0 & 1 & 0\end{array}$

$\begin{array}{llllllll}1 & 0 & 0 & 0 & 0 & 0 & 0 & 0\end{array}$

$\begin{array}{llllllll}0 & 0 & 0 & 0 & 0 & 0 & 1 & 0\end{array}$

Sulculeolaria chuni

Sulculeolaria quadrivalvis

Turritopsis nutricula

\section{Ctenophora}

Berosp.

Copepoda

Acartia clausi

Acartia dana

Acartia latisetosa

$\begin{array}{llllllll}0 & 0 & 1 & 0 & 1 & 0 & 0 & 0 \\ 0 & 0 & 0 & 0 & 1 & 1 & 0 & 0 \\ 0 & 0 & 0 & 0 & 1 & 0 & 0 & 0 \\ 0 & 0 & 0 & 0 & 1 & 0 & 0 & 0 \\ 0 & 0 & 0 & 0 & 1 & 1 & 0 & 0 \\ 1 & 1 & 1 & 0 & 1 & 0 & 0 & 0 \\ 0 & 0 & 1 & 0 & 1 & 0 & 0 & 0 \\ 0 & 0 & 1 & 1 & 0 & 1 & 0 & 0 \\ 0 & 0 & 0 & 0 & 1 & 0 & 0 & 0 \\ 0 & 0 & 0 & 0 & 0 & 1 & 0 & 0 \\ 0 & 0 & 1 & 0 & 1 & 0 & 0 & 0 \\ 0 & 0 & 0 & 0 & 0 & 1 & 0 & 0 \\ 0 & 0 & 1 & 0 & 0 & 1 & 0 & 0 \\ 0 & 0 & 0 & 0 & 1 & 1 & 0 & 0 \\ 0 & 0 & 0 & 0 & 1 & 0 & 0 & 0 \\ 0 & 0 & 0 & 0 & 0 & 1 & 0 & 0 \\ 1 & 1 & 1 & 0 & 1 & 0 & 1 & 1 \\ 0 & 0 & 1 & 0 & 1 & 0 & 0 & 0 \\ 0 & 0 & 1 & 0 & 0 & 1 & 0 & 0 \\ 0 & 0 & 1 & 0 & 0 & 0 & 0 & 0 \\ 0 & 0 & 0 & 0 & 1 & 1 & 0 & 0 \\ 0 & 0 & 1 & 0 & 0 & 0 & 0 & 0 \\ 0 & 0 & 0 & 0 & 1 & 0 & 0 & 0\end{array}$

$\begin{array}{llllllll}0 & 0 & 1 & 1 & 0 & 0 & 0 & 0\end{array}$

$\begin{array}{llllllll}0 & 1 & 1 & 1 & 1 & 1 & 1 & 1 \\ 1 & 0 & 1 & 0 & 0 & 0 & 0 & 1\end{array}$

$\begin{array}{llllllll}1 & 1 & 1 & 0 & 0 & 0 & 0 & 1\end{array}$ 


\section{ZOOPLANKTON COMMUNITY OFF THE EGYPTIAN 101 MEDITERRANEAN COAST}

Table 1-Continued

Acartia longiremis Acartia negligens

Atedias armatus

Calanus brevicornis

Calocalanus pavo

Candacea armata

Candacea bispinosa

Centropages kroyeri

Centropages typicus

Centropages violaceus

Clausocalanus arcuicornis

Clausocalanus furcatus

Clytemnestra scutellata

Copilia mediterranean

Copilia quadrata

Copilia vitra

Coyycaeus clausi

Corycaeus flaccus

Corycaeus furcifer

Corycaeus latus

Corycaeus limbatus

Corycaeus speciosus

Corycaeus typicus

Corycella carinata

Corycella rostrata

Cymbasoma rigidium

Euaetideus giesbrechti

Eucalanus atteruatus

Eucalanus elongatus

Eucalanus crassus

Euchaeta hebes

Euchaeta marina

Euchaeta spinasa

Euchirella rostrata

Euterpina acutifrons

Haloptilus longicornis

Isias clavipes

Sidi Barrani Matrouh Alamain Sahl Al-Tina

A 00 W 01 A 00 W ol A 00 W 01 A 00 A 00

$\begin{array}{llllllll}1 & 1 & 1 & 0 & 1 & 1 & 1 & 1 \\ 0 & 0 & 1 & 0 & 1 & 1 & 1 & 0 \\ 1 & 0 & 0 & 0 & 0 & 0 & 0 & 0 \\ 1 & 1 & 1 & 1 & 0 & 0 & 0 & 0 \\ 1 & 1 & 1 & 0 & 1 & 1 & 1 & 1 \\ 0 & 1 & 0 & 1 & 0 & 0 & 0 & 0 \\ 0 & 1 & 0 & 0 & 1 & 1 & 0 & 0 \\ 0 & 0 & 1 & 0 & 1 & 0 & 0 & 0 \\ 1 & 1 & 1 & 1 & 1 & 0 & 1 & 0 \\ 0 & 1 & 1 & 0 & 1 & 0 & 1 & 0 \\ 1 & 1 & 1 & 1 & 1 & 1 & 1 & 1 \\ 0 & 1 & 0 & 0 & 1 & 1 & 0 & 0 \\ 0 & 0 & 0 & 0 & 1 & 1 & 0 & 0 \\ 1 & 0 & 0 & 0 & 0 & 0 & 0 & 0 \\ 1 & 0 & 0 & 0 & 1 & 0 & 0 & 0 \\ 0 & 0 & 0 & 0 & 1 & 0 & 0 & 0 \\ 1 & 1 & 1 & 1 & 1 & 1 & 1 & 1 \\ 1 & 1 & 1 & 0 & 1 & 0 & 0 & 1 \\ 0 & 0 & 1 & 0 & 0 & 0 & 0 & 0 \\ 0 & 1 & 1 & 0 & 0 & 0 & 0 & 0 \\ 0 & 0 & 1 & 0 & 1 & 0 & 0 & 0 \\ 0 & 0 & 1 & 1 & 1 & 1 & 0 & 0 \\ 0 & 0 & 1 & 0 & 1 & 1 & 0 & 0 \\ 1 & 1 & 1 & 0 & 1 & 1 & 0 & 1 \\ 0 & 0 & 1 & 0 & 1 & 1 & 1 & 0 \\ 0 & 1 & 0 & 0 & 0 & 0 & 0 & 0 \\ 1 & 0 & 1 & 0 & 0 & 0 & 0 & 0 \\ 1 & 1 & 1 & 1 & 1 & 1 & 1 & 1 \\ 1 & 0 & 1 & 0 & 0 & 0 & 1 & 0 \\ 0 & 1 & 0 & 0 & 0 & 0 & 0 & 0 \\ 0 & 1 & 0 & 0 & 0 & 0 & 0 & 0 \\ 1 & 1 & 1 & 0 & 1 & 1 & 1 & 0 \\ 1 & 1 & 1 & 0 & 0 & 1 & 0 & 0 \\ 0 & 1 & 1 & 0 & 1 & 1 & 0 & 0 \\ 1 & 1 & 1 & 1 & 1 & 1 & 1 & 1 \\ 1 & 1 & 1 & 1 & 1 & 1 & 0 & 0 \\ & & 1 & 0 & 0 & 0 & 0 & 0\end{array}$


Table 1- Continued

Sidi Barrani Matrouh Alamain Sahl Al-Tina A 00 W of A 00 W 01 A 00 W01 A 00 A 00

Lubbokia squalimana

$\begin{array}{llllllll}0 & 0 & 0 & 0 & 1 & 0 & 0 & 1 \\ 0 & 1 & 1 & 0 & 1 & 1 & 0 & 0 \\ 0 & 0 & 1 & 0 & 0 & 0 & 0 & 0 \\ 1 & 1 & 1 & 0 & 0 & 0 & 1 & 1 \\ 1 & 0 & 1 & 0 & 1 & 1 & 0 & 0 \\ 0 & 0 & 1 & 0 & 1 & 0 & 0 & 0 \\ 1 & 1 & 1 & 1 & 1 & 1 & 1 & 0 \\ 0 & 0 & 1 & 0 & 0 & 0 & 0 & 0 \\ 0 & 0 & 1 & 0 & 0 & 1 & 0 & 0 \\ 0 & 0 & 1 & 0 & 1 & 0 & 1 & 0 \\ 0 & 0 & 1 & 0 & 1 & 1 & 0 & 0 \\ 0 & 0 & 0 & 0 & 0 & 0 & 0 & 1 \\ 0 & 0 & 0 & 0 & 1 & 1 & 0 & 0 \\ 1 & 1 & 1 & 1 & 1 & 1 & 1 & 1 \\ 1 & 1 & 1 & 1 & 1 & 1 & 1 & 1 \\ 0 & 0 & 1 & 0 & 1 & 1 & 0 & 0 \\ 0 & 0 & 1 & 0 & 1 & 1 & 1 & 0 \\ 1 & 1 & 1 & 0 & 1 & 1 & 1 & 1 \\ 0 & 1 & 0 & 0 & 0 & 0 & 0 & 0 \\ 1 & 1 & 1 & 1 & 1 & 1 & 1 & 1 \\ 0 & 0 & 1 & 0 & 1 & 1 & 1 & 0 \\ 0 & 0 & 1 & 0 & 0 & 0 & 0 & 0 \\ 0 & 0 & 0 & 0 & 1 & 0 & 0 & 0 \\ 0 & 0 & 1 & 0 & 1 & 1 & 0 & 0 \\ 0 & 0 & 1 & 0 & 1 & 1 & 0 & 0 \\ 0 & 0 & 1 & 0 & 1 & 0 & 0 & 0 \\ 1 & 0 & 1 & 0 & 0 & 0 & 0 & 0 \\ 1 & 0 & 0 & 0 & 0 & 0 & 0 & 0 \\ 0 & 0 & 0 & 0 & 1 & 0 & 0 & 0 \\ 1 & 0 & 0 & 0 & 0 & 1 & 0 & 0 \\ 1 & 0 & 1 & 0 & 1 & 1 & 1 & 1 \\ 1 & 1 & 1 & 1 & 1 & 1 & 1 & 1 \\ 1 & 0 & 1 & 1 & 1 & 1 & 1 & 0\end{array}$

Lucicutia flavicornis

Lucicutia ovalis

Macrosetella gracilis

Mecynocera clausi

Macrosetell norvigeca

Macrosetell rosea

Mormonilla phasma

Nannocalanus minor

Neocalanus gracilis

Neocalanus tenuicornis

Nitocera lacustris

Oithona linearis

Oithona nana

Oithona plumifera

Oncaea conifera

Oncaea mediterranea

Oncaea venusta

Paracalanus nanus

Paracalanus parvus

Paracalanus pygmaeus

Phaenna spinifera

Pleuromamma abdominalis

Pleuromamma gracilis

Rhinocalanus nastus

Sappharina angusta

Sappharina mechialosa

Sappharina opalina

Scolecithrix brandyi

Spinocalanus abyssalis

Temora stylifera

Nauplius larvae

Copepodite stages

\section{Cladocera}

Evadne spinifera

Evadne tergestina

Podon polyphemoides

$\begin{array}{llllllll}1 & 0 & 1 & 0 & 1 & 0 & 1 & 1 \\ 1 & 0 & 0 & 0 & 1 & 0 & 0 & 0 \\ 0 & 0 & 1 & 0 & 0 & 0 & 0 & 0\end{array}$ 
Table 1- Continued

Sidi Barrani Matrouh Alamain Sahl Al-Tina

A 00 W 01 A 00 W 01 A 00 W 01 A 00 A 00

Ostracoda

Conchoecia elegans

Conchoecia obtusata

Cypridina castania

Cypridina mediterranea

Pteropoda

Creseis acicula

Creseis virgula

Euclio pyramidata

Hyalocylis striata

Limacina helicina

Limacina inflata

Limacina trochiformis

Peraclis reticulata

Heteropoda

Firola coronata

Chaetognatha

Sagitta bipunctata

Sagitta friderici

Sagitta inflata

Sagitta macrocephala

Sagitta serratodentata

Sagitta setosa

Larvacea

Appendicularia sicula

Fritillaria borealis

Fritillaria formica

Fritillaria haplostoma

Fritillaria megachile

Oikopleura albicans

Oikopleura cophocerca

Oikopleura dioica

Oikopleura fusiformis

Oikopleura intermedia

Oikopleura longicauda

Oikopleura parva

$\begin{array}{llllllll}0 & 0 & 1 & 0 & 1 & 1 & 0 & 0 \\ 1 & 1 & 1 & 0 & 1 & 0 & 0 & 1 \\ 0 & 0 & 1 & 0 & 0 & 0 & 0 & 0 \\ 0 & 0 & 1 & 0 & 0 & 1 & 0 & 0 \\ & & & & & & & \\ 0 & 0 & 1 & 0 & 0 & 1 & 0 & 0 \\ 1 & 0 & 1 & 0 & 0 & 0 & 0 & 0 \\ 0 & 0 & 1 & 0 & 1 & 1 & 0 & 0 \\ 1 & 0 & 0 & 0 & 0 & 0 & 0 & 0 \\ 0 & 0 & 0 & 0 & 1 & 0 & 0 & 0 \\ 1 & 1 & 1 & 1 & 1 & 1 & 1 & 1 \\ 0 & 0 & 1 & 0 & 0 & 1 & 0 & 0 \\ 0 & 0 & 0 & 0 & 1 & 0 & 0 & 0\end{array}$

$\begin{array}{llllllll}1 & 0 & 1 & 0 & 0 & 0 & 0 & 0\end{array}$

$\begin{array}{llllllll}0 & 0 & 0 & 0 & 1 & 0 & 0 & 0 \\ 1 & 1 & 1 & 1 & 1 & 1 & 0 & 0 \\ 0 & 1 & 0 & 0 & 1 & 1 & 1 & 0 \\ 0 & 0 & 1 & 0 & 0 & 1 & 0 & 0 \\ 0 & 0 & 0 & 0 & 1 & 1 & 0 & 0 \\ 1 & 1 & 0 & 0 & 0 & 0 & 0 & 0\end{array}$

$\begin{array}{llllllll}0 & 0 & 1 & 0 & 1 & 0 & 0 & 0\end{array}$

$\begin{array}{llllllll}0 & 1 & 1 & 1 & 1 & 1 & 1 & 0\end{array}$

$\begin{array}{cccccccc}0 & 0 & 0 & 0 & 0 & 1 & 0 & 0\end{array}$

$\begin{array}{llllllll}0 & 1 & 0 & 0 & 0 & 0 & 0 & 0\end{array}$

$\begin{array}{llllllll}1 & 0 & 1 & 0 & 1 & 0 & 0 & 0\end{array}$

$\begin{array}{llllllll}0 & 1 & 0 & 0 & 0 & 0 & 0 & 0\end{array}$

$\begin{array}{llllllll}1 & 0 & 1 & 0 & 0 & 1 & 0 & 0\end{array}$

$\begin{array}{llllllll}1 & 0 & 1 & 1 & 1 & 1 & 1 & 0\end{array}$

$\begin{array}{llllllll}1 & 0 & 1 & 0 & 0 & 0 & 0 & 0\end{array}$

$\begin{array}{llllllll}0 & 1 & 1 & 1 & 1 & 1 & 0 & 0\end{array}$

$\begin{array}{llllllll}1 & 1 & 1 & 1 & 1 & 1 & 1 & 1\end{array}$

$\begin{array}{llllllll}1 & 1 & 1 & 1 & 1 & 1 & 1 & 0\end{array}$ 
Table 1 - Continued

Sidi Barrani Matrouh Alamain Sahl Al-Tina A 00 W $01 A 00 W 01$ A 00 W01 A 00 A 00

Pegalopleura haranti

Polychaete sp.

Free livina Nematodes

Decapoda

Leucifer acestra

Euphausiacea

Stylocheiron sp.

Mysidacea

Mysid sp.

Amphipoda

Hyperia sp.

Pseudolyceae sp.

Thaliacea

Doliolum denticulatum

Doliotella gegenbauri

Salpa fusiformis

Salpa maxima

Thalia democratica

$$
A=\text { Autumn, } W=\text { Winter }
$$

$\begin{array}{llllllll}0 & 0 & 0 & 0 & 1 & 1 & 0 & 0 \\ 1 & 0 & 1 & 0 & 1 & 0 & 0 & 1 \\ 0 & 0 & 0 & 0 & 0 & 0 & 1 & 1 \\ 1 & 0 & 1 & 0 & 1 & 1 & 1 & 0 \\ 1 & 0 & 1 & 0 & 1 & 1 & 1 & 0 \\ 1 & 1 & 1 & 0 & 0 & 0 & 0 & 0 \\ 1 & 1 & 1 & 0 & 1 & 0 & 0 & 0 \\ 1 & 0 & 1 & 0 & 1 & 1 & 0 & 0 \\ 1 & 0 & 1 & 0 & 1 & 1 & 0 & 1 \\ 1 & 0 & 1 & 0 & 0 & 0 & 0 & 1 \\ 1 & 0 & 0 & 0 & 1 & 1 & 0 & 0\end{array}$

$\begin{array}{llllllll}0 & 1 & 1 & 0 & 1 & 0 & 0 & 0\end{array}$

$\begin{array}{llllllll}0 & 0 & 1 & 0 & 0 & 0 & 0 & 0\end{array}$

$\begin{array}{llllllll}1 & 0 & 1 & 0 & 0 & 0 & 0 & 0\end{array}$

$\begin{array}{llllllll}0 & 0 & 0 & 0 & 0 & 1 & 0 & 0\end{array}$

$\begin{array}{llllllll}0 & 1 & 0 & 0 & 0 & 0 & 0 & 0\end{array}$ 


\section{ZOOPLANKTON COMMUNITY OFF THE EGYPTIAN 105 MEDITERRANEAN COAST}

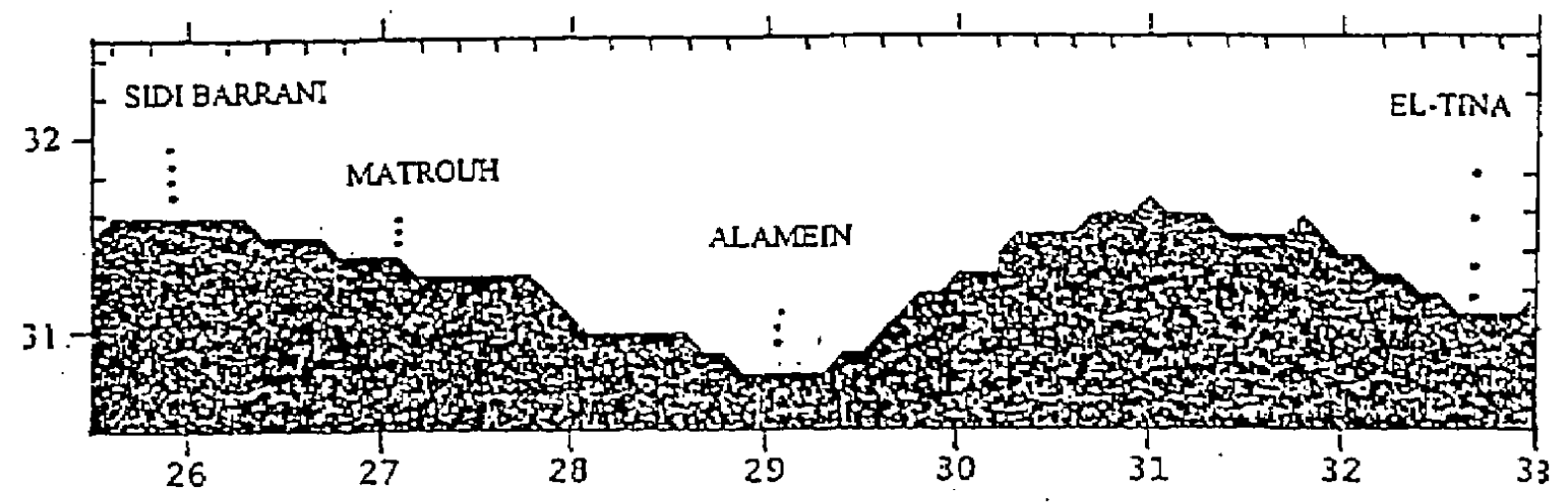

Fig.1-Study area off the Egyptian Mediterranean Waters and Sampling Positions

国 Total copepods m Other groups 日Meroplankton
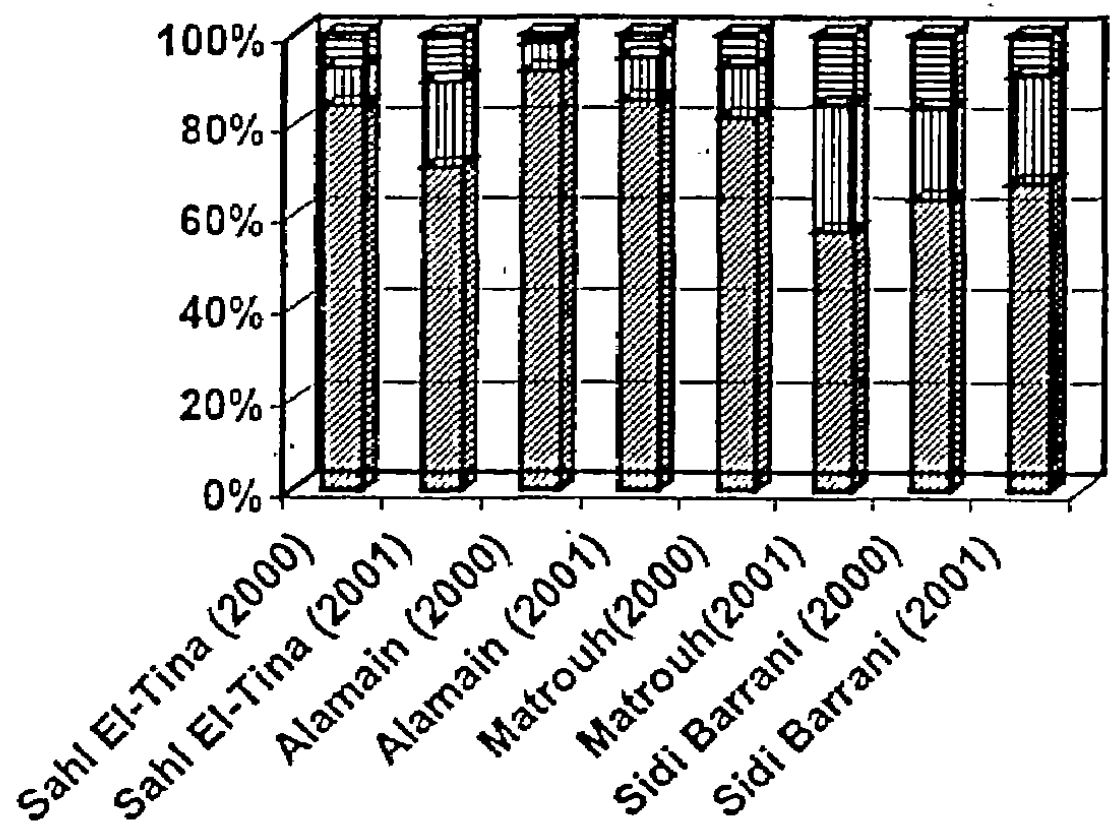

1. 2- Relative abundance of zooplankton groups along the Egyptian Mediterranean Co 


\section{Nagwa, E.M. Abdel-Aziz and Sawsan M. Aboul-Ezz}

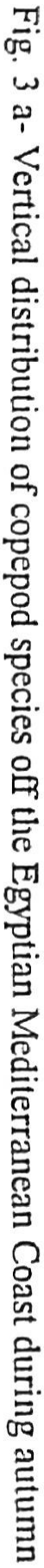

J55

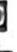

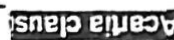

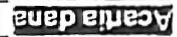

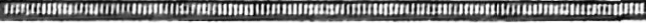

Mrandminturin

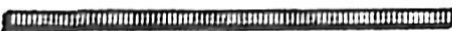

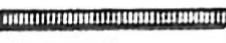

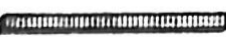

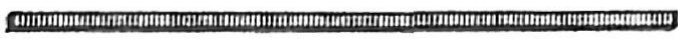

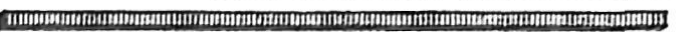

ก

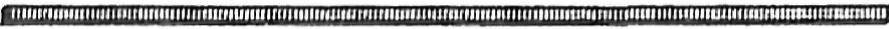

(m)

mentmm

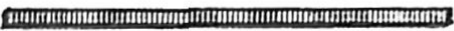

1

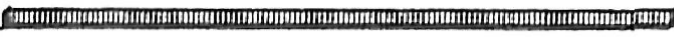

C

का

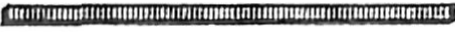

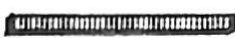

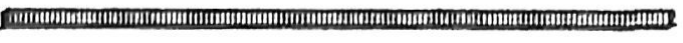

A

ल

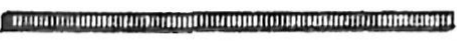

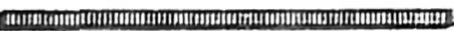

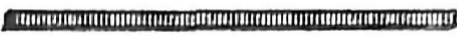

-

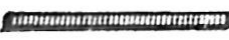

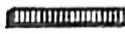

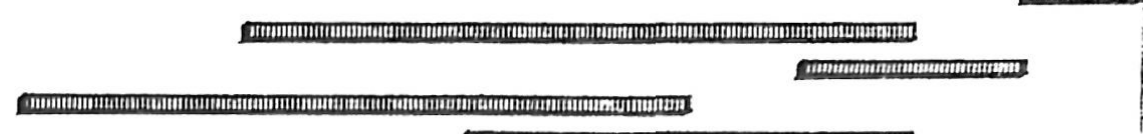

ค1

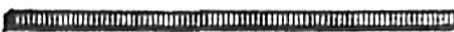

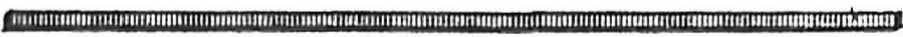

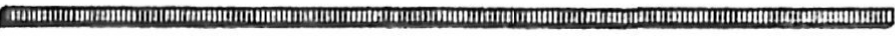

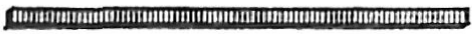

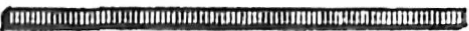

(1)

-

ค

A

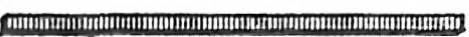

1.

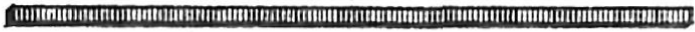

(1)

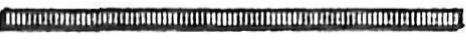

С

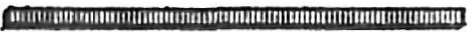

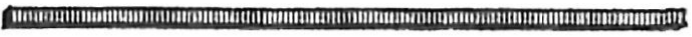

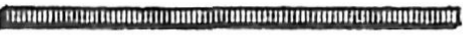

(1)

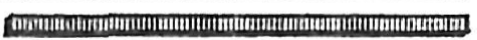

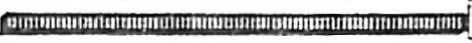

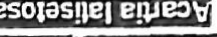

जण्यांगण हायए:

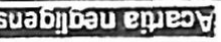

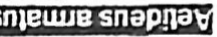

जाण्यामश्यव Snण

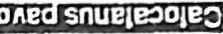

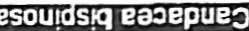

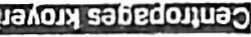

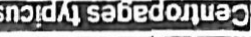

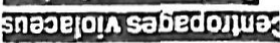

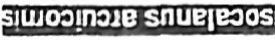

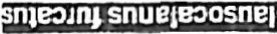

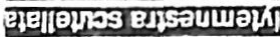

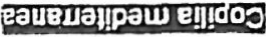

सांग्यासक दाएक्⿹

ब्याजदाएश

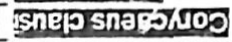

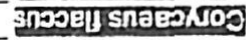

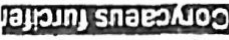

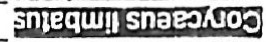

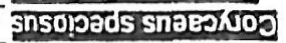

जIII

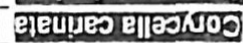

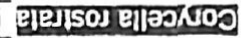

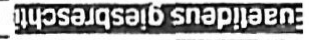

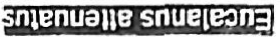

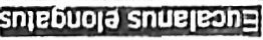

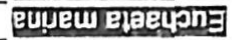

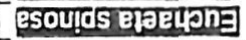

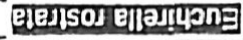

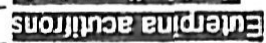

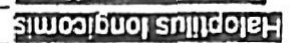

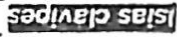

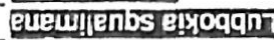

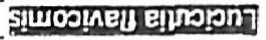

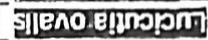

ज्ञाएस

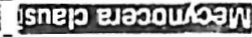

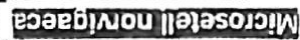

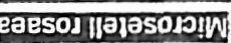

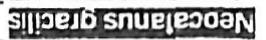

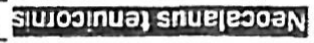

ज्ञास्या हमण्याए०

हपरण हण प्याए

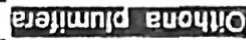

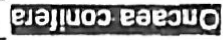

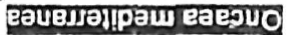

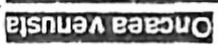

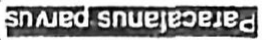

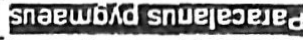

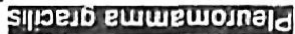

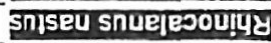

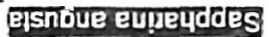

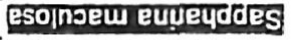

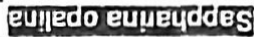

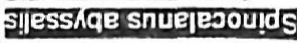

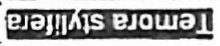




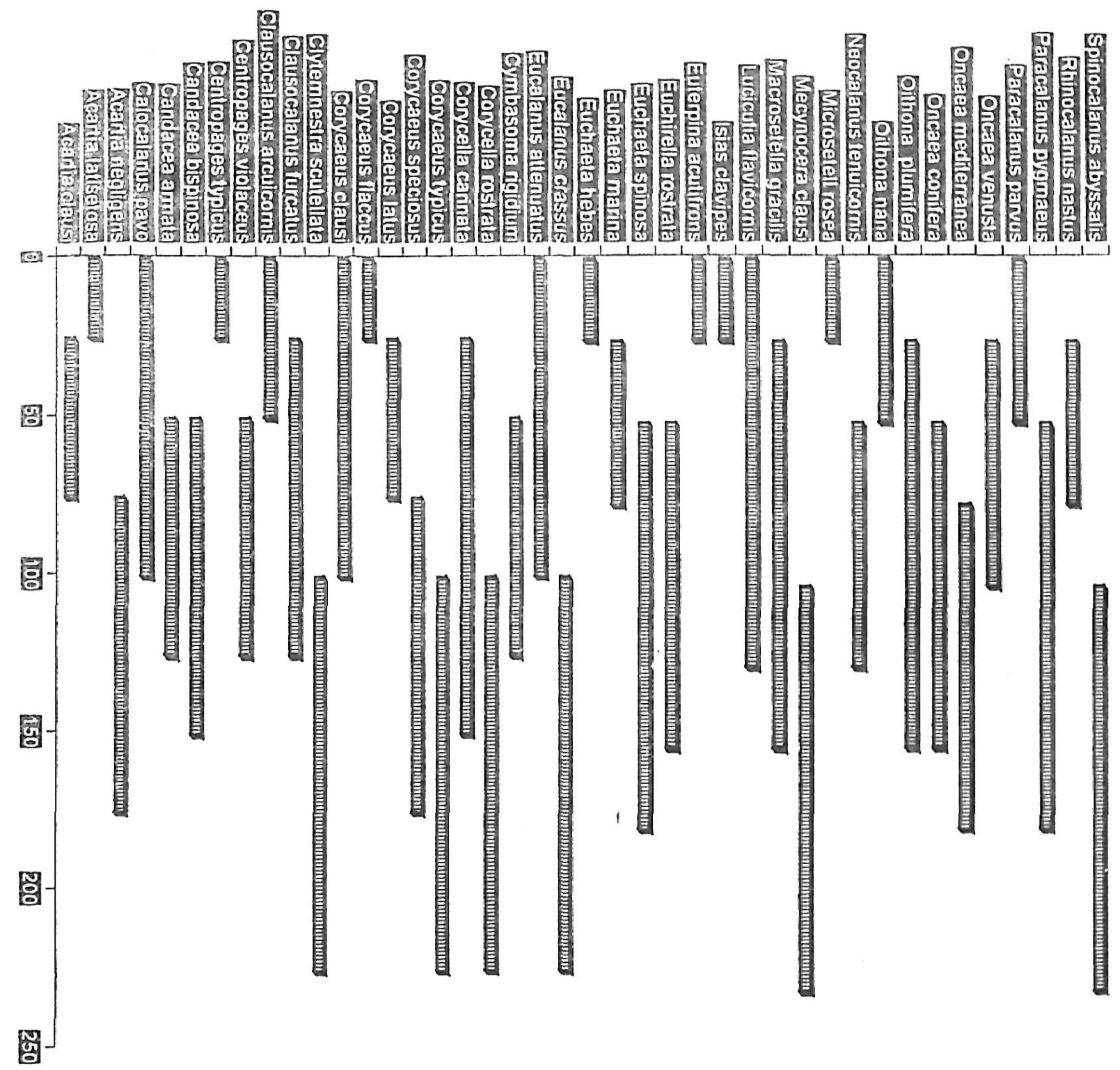

Fig. 3b- Vertical distribution of copepod species off the Egyptian Mediterranean Coast during winter 